\title{
Possible Explanation for Cancer in Rats due to Cell Phone Radiofrequency Radiation
}

\author{
Bernard J. Feldman \\ University of Missouri-St. Louis, St. Louis, USA
}

\begin{abstract}
Very recently, the National Toxicology Program reported a correlation between exposure to whole body $900 \mathrm{MHz}$ radiofrequency radiation and cancer in the brains and hearts of Sprague Dawley male rats. Assuming that the National Toxicology Program is statistically significant, we propose the following explanation for the results. The neurons around the brain and heart form closed electrical circuits and, following Faraday's Law, $900 \mathrm{MHz}$ radiofrequency radiation induces $900 \mathrm{MHz}$ electrical currents in these neural circuits. In turn, these $900 \mathrm{MHz}$ currents in the neural circuits generate sufficient localized heat in the neural cells to shift the equilibrium concentration of carcinogenic radicals to higher levels, and thus, to higher incidences of cancer. These ideas are then applied to the question of the risk to humans from cell phone radiofrequency radiation.
\end{abstract}

Keywords: cancer, cell phone radiofrequency radiation, rats, mice, Faraday’s Law, schwannomas, gliomas, neural circuits

\section{Introduction}

The question whether cell phone radiofrequency radiation causes cancer is of great interest to virtually every student, given the incredible high percentage of students who use cell phones numerous times every day. This paper summarizes the very recent and most conclusive results on this question and then presents a simple theory to explain these results, using only freshman level science. In particular, the only scientific ideas used in the explanation are Faraday's Law and Ohm's Law from physics, the law of mass action from chemistry, and physiological facts from biology. This material would be appropriate for most high school, college, and university introductory science classes. There is also a brief discussion of the risk to humans from cell phone radiofrequency radiation.

Very recently, the National Toxicology Program (Wyde et al., 2016) reported a correlation between exposure to whole body radiofrequency radiation and cancer in Sprague Dawley rats. The experiment consisted of irradiating Sprague Dawley rats and B6C3F1 mice with $900 \mathrm{MHz}$ radiation with four different intensity exposures: $0 \mathrm{~W} / \mathrm{kg}, 1.5 \mathrm{~W} / \mathrm{kg}, 3 \mathrm{~W} / \mathrm{kg}$, and $6 \mathrm{~W} / \mathrm{kg}$. The frequency of $900 \mathrm{MHz}$ is typical for use in cell phones and other wireless devices. The exposures were 10 mins. on and 10 mins. off for 18 hours a day, resulting in a total exposure of nine hours daily. The animals were exposed daily from in utero until two years of age. The animals were monitored so that exposure was at a low non-thermal or non-heating level. Groups of 90 animals were used for each species, sex, and intensity.

They reported the following results:

Bernard J. Feldman, Dr., professor, Department of Physics and Astronomy, University of Missouri-St. Louis. 
1. Excess cancers were found only in male rats but not in female rats, male mice, or female mice;

2. Only brain cancers (gliomas and brain lesions) and heart cancers (schwannomas) were found. Schwannomas are cancers of the neural cell sheaths;

3. The incidence of cancer in male rats increased as the $900 \mathrm{MHz}$ radiofrequency intensity increased from 0 to $6 \mathrm{~W} / \mathrm{kg}$. In particular, no cancers were found in any animal that was not exposed to radiofrequency radiation $(0 \mathrm{~W} / \mathrm{kg})$;

4. Even at the highest radiofrequency power, $6 \mathrm{~W} / \mathrm{kg}$, this power was insufficient to increase the rats' average body temperature by more than one degree centigrade;

5. The rats exposed to radiofrequency radiation lived longer than those rats that were not exposed;

6. Analysis of bioassays showed that "male rats are more sensitive to chemical carcinogenesis compared to female rats;”

7. A small minority of the reviewers of this study questioned the statistical significance of these results. One of the authors also mentioned that previous studies by other groups had found similar brain and heart excess cancers in humans due to radiofrequency radiation (Melnick, 2016).

\section{Previous Work}

Theoretical understanding of the interaction between animals and electromagnetic radiation has a long and complicated history. Physicists, in general, have been very skeptical of any connection between any non-ionizing radiation and cancer. The classic paper by Adair (1992) on weak extremely-low-frequency (60 Hz) electromagnetic fields concluded that "such interactions are too weak to have a significant effect on human biology at the cell level.” Adair applied Faraday's Law to a cell radiated with weak $60 \mathrm{~Hz}$ electromagnetic fields and concluded that the induced electric field is small compared to thermal noise induced electric fields. Even this author expressed skepticism about cell phone radiation (900 MHz) causing cancer by using an analogy with Einstein's theory of the photoelectric effect-the $900 \mathrm{MHz}$ photon energies are about a million times less than the energy needed to break chemical bonds (Feldman, 2014). Recently, Barnes and Greenebaum (2016) proposed that weak static and high frequency magnetic fields could change the recombination rate of radical pairs, and thus, change the concentration of carcinogenic radicals like $\mathrm{O}_{2}^{-}$in cells. Panagopoulos, Johansson, and Carlo (2015) suggested that high frequency electric fields exert electrostatic forces on the cell membrane, disrupting the functioning of the ion channels.

\section{Theoretical Model}

Assuming that the National Toxicology Program is statistically significant, the following explanation for the results is proposed. The neurons around the brain and heart form closed electrical circuits and, following Faraday’s Law, $900 \mathrm{MHz}$ radiofrequency radiation induces $900 \mathrm{MHz}$ electrical currents in these neural circuits. Given that these neural circuits could be one neuron in width and neuron axons are about one micrometer in diameter, these $900 \mathrm{MHz}$ currents in the neural circuits could generate sufficient localized heat in the neural cells to significantly raise the temperature of the neural and neighboring cells and shift the equilibrium concentration of carcinogenic radicals in these cells to higher levels and thus, to higher incidences of cancer.

Consider a neural circuit on the surface of the brain or the heart in the shape of a circle of radius $r$. From Faraday's Law, the induced voltage, $V$, in the neural circuit is equal to minus the time $(t)$ derivative of the 
magnetic flux crossing the closed circuit. Assuming that the radiofrequency magnetic field is $B=B_{0} \cos (\omega t)$, where $B_{0}$ is a constant and $\omega / 2 \pi=900 \mathrm{MHz}$, then $V=\omega \mathrm{B}_{0} \sin (\omega t) \pi r^{2}$ and is proportional to $\mathrm{B}_{0}, r^{2}$, and $\omega$. The resistance of the neural circuit, $R$, is proportional to its circumference, and thus, proportional to $r$, since the size of neural cells is largely independent of the size of the animal. Then, by Ohm's Law, the heat generated per second (power) in the neural circuit, $P=V^{2} / R$, and is proportional to $\mathrm{B}_{0}{ }^{2}, \omega^{2}$, and $r^{3}$, and the heat generated per second per unit length of the neural circuit is proportional to $\mathrm{B}_{0}{ }^{2}, \omega^{2}$, and $r^{2}$.

Now question arises-How could this excess localized heat cause cancer? Again, let us consider a simple model consisting of carcinogenic radicals like oxidants, $\mathrm{O}$, and antioxidants that scavenge these radicals, A. Inside cells, chemical reactions occur that convert food into useful chemicals, heat, and muscular motion. Carcinogenic radicals are created as waste products. In order for the animal to protect itself from these carcinogenic radicals, the cells create antioxidants.The antioxidants bind to the carcinogenic radicals forming harmless molecules, OA, which then diffuse to nearby veins and are removed from the body via the kidneys and urinary system.The concentrations of $\mathrm{O}$ and $\mathrm{A}$ are in approximate equilibrium with $\mathrm{OA}$, namely, the cell's rate of production of $\mathrm{O}$ equals the cell's rate of production of $\mathrm{A}$, which in turn equals the rate of removal of OA from the cell. One can write down the following chemical reaction:

$$
\mathrm{O}+\mathrm{A}=\mathrm{OA}
$$

From the law of mass action, at equilibrium, the concentrations of O ([o]), A ([a]), and OA ([oa]), are related to an equilibrium constant $\mathrm{K}$ that depends of temperature.

$$
\begin{gathered}
{[\mathrm{oa}] /[\mathrm{o}][\mathrm{a}]=\mathrm{K}(\mathrm{T})} \\
\mathrm{K}(\mathrm{T})=\mathrm{C} \exp (-\mathrm{G} / \mathrm{RT})
\end{gathered}
$$

Here, $\mathrm{C}$ is a constant, $\mathrm{T}$ is the temperature in Kelvin, $\mathrm{G}$ is the Gibbs free energy of the chemical reaction, and $\mathrm{R}$ is the gas constant. $G$ is negative for exothermic reactions. Notice as the temperature goes up, K(T) goes down, and [o] and [a] go up. An increase in [o] is believed to be connected with an increase in the cancer rate.

An alternative possibility is that the increased temperature denatures antioxidants (they lose their structure and thus their ability to function), leaving higher concentrations of carcinogenic radicals. In humans, proteins start to denature when the body temperature is above $42{ }^{\circ} \mathrm{C}$, causing death; this temperature is only $5{ }^{\circ} \mathrm{C}$ above the human body temperature of $37^{\circ} \mathrm{C}$.

\section{Comparison Between Model and Experiments}

This model is reasonably consistent with the National Toxicology Program's experimental observations. The cancers are found near neurons and in large organs that are surrounded by or largely consist of neural cells, like the brain and the heart, but not found in small organs, like the thyroid, prostate, or the kidney or in large organs not surrounded by or consisting of neural cells, like the pancreas, lung, or the liver. The incidence of cancer increased with the radiofrequency intensity, which is proportional to $\mathrm{B}_{0}{ }^{2}$. Even though the rats' average body temperature does not increase significantly, there could be significant localized heating of these neural circuits undetected by the National Toxicology Program. Given that male Sprague Dawley rats are on average about $60 \%$ heavier (and thus about 60\% larger) than female Sprague Dawley rats at 15 weeks of age, the model does predict a higher incidence of cancer in male rats than female rats, but the increased sensitivity of male rats to chemical carcinogenesis probably plays a much bigger role in this difference.

This model is also consistent with the observation that no cancers were found in mice. Since mice are 
about a factor of three smaller in size than the Sprague Dawley rats, our theory predicts that the incidence of cancer should be reduced by a factor of 9 . Given that the incidence of cancer in male rats was barely statistically significant, a factor of 9 would predict no cancer observed in male mice. This observation of no cancer in male mice strongly argues against any cancer mechanism that is at the cellular or molecular level, like those suggested by Barnes and Greenebaum (2016) and Panagopoulos, Johansson, and Carlo (2015). Given that nerve cells are about the same size in mice and rats and the genetics and biology of mice and rats are extremely similar, a cellular or molecular theory of cancer would predict cancer in male mice, if there is cancer in male rats. Finally, this model is consistent with a recent article by Burlaka et al. (2013), reporting the overproduction of free radicals in quail embryonal cells exposed to cell phone radiation.

Finally, the National Toxicology Report did comment on the observation that irradiated rats lived longer than non-irradiated rats (Wyde et al., 2016). They suggested that it is related to the observation that calorie-restricted animals live longer on average. The radiofrequency radiation that is absorbed by rats generates heat throughout their bodies, thus leading to less consumption of food and less production of carcinogenic radicals.

\section{Model Predictions}

This model makes some predictions. Given that the male Sprague Dawley rats at age 15 weeks range in weight (and thus size) from 320-460 grams, this model predicts that the larger male rats should have a higher incidence of cancer than the smaller male rats. This model also predicts that the incidence of cancer should increase as the square of the radio wave frequency, assuming that the resistance of the neural circuit does not change with frequency. It is clear that this mechanism is not relevant to $60 \mathrm{~Hz}$ electromagnetic fields, given that $60 \mathrm{~Hz}$ is so much smaller than $900 \mathrm{MHz}$.

Finally, how are these ideas connected with the relationship between cell phone radiation and cancer in humans? At first glance, one might conclude that the incidence of cancer due to cell phone radiation should be much greater in humans than in rats, because our hearts and brains are so much bigger. Let me caution against such simple logic for the following reasons:

1. Rats are not perfect analogues to humans. There are diseases found in rats that are not found in humans and visa versa;

2. The rats were exposed to radiation nine hours a day from utero to the time they were killed, while the exposure in humans is much less;

3. The radiation actually increased the lives of the rats. If the same is true in humans, many human autopsies would not be looking for these types of cancers.

The question of cancer in humans due to radiofrequency radiation must ultimately be determined by epidemiologic studies on humans. A very recent publication by Wojcik (2016) on a meta-analysis of many different human epidemiologic studies reported that the risk for glioma increased by a factor of 1.9 for cell phone users; it also mentioned the French Cerent collaborative case-control study that showed the risk for glioma increased by a factor of 2.89 for heavy cell phone users. Clearly, our students should be made aware of this risk and hopefully, the inclusion of this material in their science classes will further that awareness. 


\section{References}

Adair, R. K. (1992). Constraints on biological effects of weak extremely-low-frequency electromagnetic fields. Physical Review A, 43, 1039-1048.

Barnes, F., \& Greenebaum, B. (2016). Some effects of weak magnetic fields on biological systems. IEEE Power Electronics Magazine, March, 60.

Burlaka, A., Tsybulin, O., Sidorik, E., Lukin, S., Polishuk, V., Tsehmistrenko, S., \& Yakymenko, I. (2013). Overproduction of free radical species in embryonal cells exposed to low intensity radiofrequency radiation. Experimental Oncology, 35, 219-225.

Feldman, B. (2014). Physics déjà vu. The Physics Teacher, 52, 391.

Melnick, R. (2016). Cell phone radiation boosts cancer rates in animals; $\$ 25$ million NTP study finds brain tumors. Microwave News. Retrieved May 25, 2016, from http://microwavenews.com/news-center/ntp-cancer-results

Panagopoulos, D., Johansson, O., \& Carlo, G. (2015). Polarization: A key difference between man-made and natural electromagnetic fields, in regard to biological activity. Retrieved October 12, 2015, from http://www.Nature.com/Scientific Reports 5:14914

Wojcik, D. (2016). Primary brain tumors and mobile phone usage. Cancer Epidemiology, 44, 123-124.

Wyde, M., Cesta, M., Blystone, C., Elmore, S., Foster, P., ... \& Bucher, J. (2016). Report of partial findings from the National Toxicology Program carcinogenesis studies of cell phone radiofrequency radiation in Hsd: Sprague Dawley SD rats (whole body exposures). Retrieved May 26, 2016, from http://biorxiv.org/content/early/2016/05/26/055699.full.pdf and https://ecfsapi.fcc.gov/file/10011773529766/EHTrustNTP.pdf 